\title{
Stabilized Skyrmion Phase Detected in MnSi Nanowires by Dynamic Cantilever Magnetometry
}

\author{
A. Mehlin, ${ }^{\dagger}$ F. Xue, ${ }^{* \dagger,}$ D. Liang ${ }^{\S}$ H. F. Du, ${ }^{\ddagger}$ M. J. Stolt, ${ }^{\S}$ S. Jin, ${ }^{*}{ }^{\S}$ M. L. Tian, ${ }^{\ddagger}$ and M. Poggio ${ }^{*}$, \\ ${ }^{\dagger}$ Department of Physics, University of Basel, Klingelbergstrasse 82, 4056 Basel, Switzerland \\ ${ }^{\ddagger}$ High Magnetic Field Laboratory, Chinese Academy of Science, Shushanhu Road 350, Hefei 230031, Anhui, P. R. China \\ ${ }^{\S}$ Department of Chemistry, University of Wisconsin-Madison, 1101 University Avenue, Madison, Wisconsin 53706, United States
}

\section{Supporting Information}

ABSTRACT: Using dynamic cantilever magnetometry we measure an enhanced skyrmion lattice phase extending from around $29 \mathrm{~K}$ down to at least $0.4 \mathrm{~K}$ in single $\mathrm{MnSi}$ nanowires (NWs). Although recent experiments on two-dimensional thin films show that reduced dimensionality stabilizes the skyrmion phase, our results are surprising given that the NW dimensions are much larger than the skyrmion lattice constant. Furthermore, the stability of the phase depends on the orientation of the NWs with respect to the applied magnetic field, suggesting that an effective magnetic anisotropy, likely due to the large surface-to-volume ratio of these nanostructures, is responsible for the stabilization. The compatibility of our technique with nanometer-scale
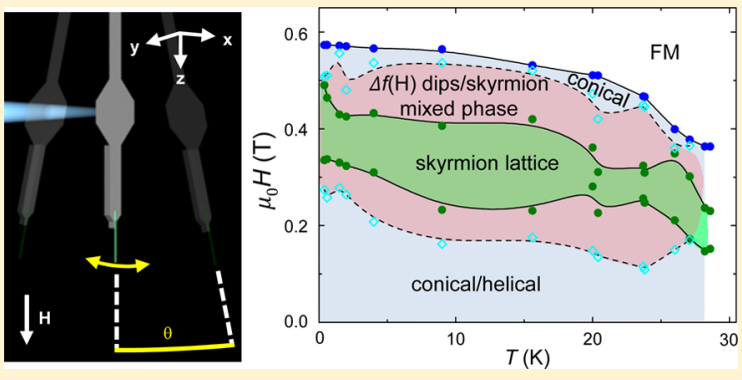
samples paves the way for future studies on the effect of confinement and surfaces on magnetic skyrmions.

KEYWORDS: Skyrmion, cantilever magnetometry, MnSi, nanowire, helimagnets

$\mathrm{M}$ agnetic skyrmions are topologically nontrivial spin configurations that appear in B20 materials with a helical ground state and arise due to Dzyaloshinskii-Moriya (DM) interactions. ${ }^{1-3}$ Since their initial observation in $2009,{ }^{4-6}$ they have been considered promising as carriers of information in high density magnetic media due to a number of favorable properties, including their stability, nanometer-scale size, and the ultralow electrical current density required to move them. ${ }^{7}$ The threshold for moving these vortex-like spin configurations is around $10^{6} \mathrm{~A} / \mathrm{m}^{2}$ compared the $10^{12} \mathrm{~A} / \mathrm{m}^{2}$ required to move a domain wall, ${ }^{8}$ allowing potential skyrmionbased memory devices to count on negligible ohmic heating. Nevertheless, since the skyrmion phase in bulk helimagnets is confined to a small region of temperature and magnetic field, the possibility for applications remains limited.

Recently, however, reduction of sample dimensions from bulk to two-dimensional (2D) thin films has been shown to expand this phase, ${ }^{9-13}$ either because of spatial confinement ${ }^{14}$ or uniaxial distortion effects. ${ }^{15}$ In this study, we use dynamicmode cantilever magnetometry $(\mathrm{DCM})^{16}$ to investigate a natural follow-up question: what is the extent of the skyrmion phase in magnetic nanowires (NWs)? Interest in these magnetic nanostructures, aside from the increased degree of confinement that they provide compared to thin films, is driven by their obvious potential for encoding information in high densities and for transmitting information from one location to another. NWs play a prominent role in nonvolatile magnetic memory proposals such as the so-called "racetrack" design based on the motion of magnetic domain walls. ${ }^{17}$ Furthermore, they are preferable to bulk material due to the reduced presence of stacking faults in their crystalline structure.

The single-crystal MnSi NWs investigated here are grown by chemical vapor deposition $(\mathrm{CVD})^{18}$ and were previously studied in a thinned-down form by Lorentz transmission electron microscopy (LTEM) ${ }^{19}$ and later through magnetoresistance measurements. ${ }^{20}$ While a measurement of NW magnetization would directly probe the skyrmion phase transition, conventional techniques such as superconducting quantum interference device (SQUID) magnetometry are not sensitive enough to measure the magnetic moment of a single NW. Measurements of ensembles are complicated by the random orientation and varying size of the $\mathrm{NWs}^{21}$ and by the presence of polycrystalline films and other morphologies of $\mathrm{MnSi}$ on the Si growth substrate.

DCM, however, is an ideal method for investigating the magnetization of individual nanostructures in defined magnetic field orientations. ${ }^{22-24}$ Here we use this sensitive technique to measure the extent of the skyrmion lattice phase in individual $\mathrm{MnSi}$ NWs. By mounting a single MnSi NW on the end of an ultrasensitive $\mathrm{Si}$ cantilever and measuring shifts in the cantilever's resonant frequency as a function of temperature, applied magnetic field, and orientation, we determine the nanostructure's magnetic phase diagram. These shifts result from the magnetic torque produced by the NW's net magnetization $\mathbf{M}$ and an externally applied magnetic field $\mathbf{H}$.

Received: June 6, 2015

Published: June 22, 2015 
Measurements are performed with the long axis of the $\mathrm{MnSi}$ NWs oriented either parallel or perpendicular to the applied field as illustrated in Figure 1. Crucially, our noninvasive
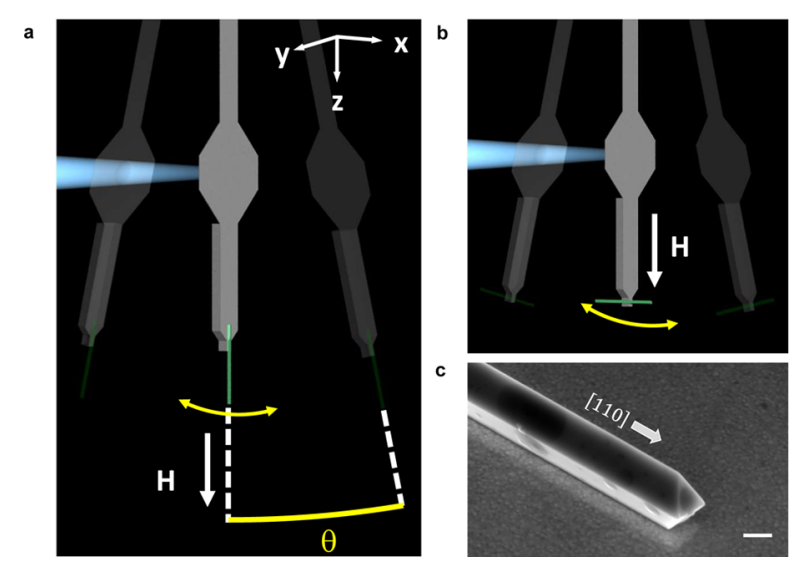

Figure 1. Schematic of the experimental setup. A MnSi NW (green) is attached to the end of an Si cantilever (gray), whose long axis is either (a) parallel or (b) perpendicular to the applied magnetic field $\mathbf{H}$. The cantilever oscillates in the $x$-direction. Laser light from the fiber interferometer is shown in white. (c) Scanning electron micrograph (SEM) of a single-crystal MnSi NW with a [110] growth direction ${ }^{18}$ placed on a gold surface. All MnSi surfaces are $\{111\}$. The scale bar is $200 \mathrm{~nm}$.

technique does not require thinning the sample to a $2 \mathrm{D}$ slab as in LTEM or making electrical contacts as in magnetoresistance or topological Hall effect (THE) measurements.

As a result of the different mechanism behind DCM compared to previously used techniques, it can provide both complementary and, as in this case, new information about skyrmion phases in nanostructures. In particular, we find strong evidence for a skyrmion lattice phase stabilized depending on the applied magnetic field orientation. This stabilization occurs despite the fact that the dimensions of the NW are too large to confine the skyrmion lattice. There is, however, an important difference between these NWs and bulk single-crystal $\mathrm{MnSi}$ samples: the NWs have an especially large surface-to-volume ratio for surfaces perpendicular to the long axis. For this reason, we hypothesize that an effective uniaxial anisotropy, likely due to the demagnetization influence of the surfaces, suppresses the alternative conical phase and favors the skyrmion configuration. $^{25,26}$

The MnSi NWs are grown along $\langle 110\rangle$ and have smooth $\{111\}$ surfaces. Their perfect B20 structure is confirmed by transmission electron microscopy (TEM) diffraction of identically grown samples. ${ }^{18}$ The cross-section of the NWs is a parallelogram with a width of about $470 \mathrm{~nm}$ as determined by scanning electron microscopy (see Figure 1c and Supporting Information). This cross-section is split by a merohedral twinning plane, specifically the (001) plane parallel with the $\langle 110\rangle$ growth direction, which partitions the NW into two parts with opposite handedness. Such twinning is expected for B20 silicide NWs and was previously observed in FeSi NWs. ${ }^{27}$

Each NW was attached to an ultrasensitive Si cantilever with epoxy (Gatan G1) using precision micromanipulators under an optical microscope. Cantilever magnetometry measurements are performed in a vacuum chamber with a pressure below $10^{-6}$ mbar at the bottom of a ${ }^{3} \mathrm{He}$ cryostat. An external magnetic field along the cantilever axis up to $|H|=6 \mathrm{~T}$ can be applied with a superconducting magnet. The single-crystal Si cantilevers are $180 \mu \mathrm{m}$ long, $4 \mu \mathrm{m}$ wide, and $0.1 \mu \mathrm{m}$ thick with a $18 \mu \mathrm{m}$ long and $1 \mu \mathrm{m}$ thick mass at the tip. Next to the mass-loaded tip is a $12 \mu \mathrm{m}$ wide paddle, which serves as a reflective surface in a fiber interferometer used for the detection of the cantilever motion. ${ }^{28}$ Interferometry is done with $100 \mathrm{nW}$ of $1550 \mathrm{~nm}$ laser light from a temperature-tunable laser diode. The quality factors of the Si cantilevers are $4 \times 10^{4}$ at $T=4 \mathrm{~K}$ and are determined by the ring-down method. The fundamental mechanical resonance frequencies of the cantilevers are between $f_{0}=2.0$ and $2.1 \mathrm{kHz}$, and their spring constants are between $k_{0}=30$ and $50 \mu \mathrm{N} / \mathrm{m}$ as determined from measurements of thermal motion at various temperatures (see Supporting Information for cantilever properties). During the measurements, the interferometric cantilever deflection signal is fed through a field programmable gate array (FPGA) circuit back to a piezoelectric element, which is mechanically coupled to the cantilever. In this way, we are able to selfoscillate the cantilever at its resonance frequency and at a desired amplitude $x_{r m s} \simeq 10 \mathrm{~nm}$ for which $\theta_{r m s} \ll 1$. Selfoscillation allows fast and accurate measurement of the cantilever's resonance frequency.

The energy of our NW-on-cantilever system can be described by the sum of a mechanical energy term, related to the cantilever (approximated here as a simple harmonic oscillator), and a magnetic energy term, related to the attached sample

$$
E=\frac{1}{2} k_{0}\left(l_{e} \sin \theta\right)^{2}+E_{m}
$$

where $k_{0}$ is the spring constant, $l_{e}$ is the effective length of the cantilever, $\theta$ is the angle of the cantilever free-end with respect to $\mathbf{H}$, and $E_{m}$ is the magnetic energy. Given that the Si cantilever and the epoxy used to attach the sample have no magnetic response, the magnetic energy depends only on the properties of the attached NW. The torque acting on the cantilever is then given by $\tau=-\partial E / \partial \theta$. Since $\theta \ll 1$ during the measurement, we can expand $E_{m}$ as a function of $\theta$ around $\theta=$ 0 . Keeping only terms up to first order in $\theta$, we solve for the cantilever's frequency shift $\Delta f=f-f_{0}$, where $f$ is the measured resonance frequency and $f_{0}$ is the resonance frequency at $H=0$

$$
\Delta f=\frac{f_{0}}{2 k_{0} l_{e}^{2}}\left(\left.\frac{\partial^{2} E_{m}}{\partial \theta^{2}}\right|_{\theta=0}\right)
$$

where $\left.\left(\left(\partial^{2} E_{m}\right) /\left(\partial \theta^{2}\right)\right)\right|_{\theta=0}$ is the second derivative of the magnetic energy with respect to $\theta$ at the cantilever's equilibrium angle (see Supporting Information for full derivation). Therefore, measurements of $\Delta f$ reveal the curvature of the magnetic energy with respect to the sample angle. Such information can shed light on the magnetic anisotropy of the sample and, under certain conditions, on the magnitude and direction of the sample's integrated magnetization $\mathbf{M}$. By focusing on the sample's average magnetic response, we can then deduce the type of magnetic configuration within and therefore its spatial dependence. Here we assume that the sample's net magnetization magnetizes along the applied field, i.e., $\mathbf{M} \| \mathbf{H}$, and taking into account shape anisotropy, we can express $M$ as a function of $\Delta f$

$$
M=l_{e} \sqrt{\frac{2 \Delta f k_{0}}{f_{0} \mu_{0} V\left(D_{\perp}-D_{\|}\right)}}
$$


where $\mu_{0}$ is the permeability of free space, $V$ is the sample volume, and $D_{\|}\left(D_{\perp}\right)$ is the demagnetization factor along (perpendicular to) the cantilever's long axis (see Supporting Information for full derivation). Note that the net magnetization $M$ is the integral of the spatially varying magnetization over the entire sample.

This description and thus eq 3 apply to magnetometry measurements made on a single $\mathrm{MnSi} \mathrm{NW}$ with its long axis aligned parallel to $\mathbf{H}$, as shown in Figure 1a. This NW, which we label NW1, is a $7.1 \mu \mathrm{m}$ long segment of a longer NW with a width of $470 \mathrm{~nm}$. Given NW1's high aspect ratio and its orientation, shape anisotropy ensures that $\mathbf{M}$ magnetizes along H. By measuring $\Delta f$ and applying eq 3 , we plot $M$ as a function of $H$, e.g., in Figure $3 \mathrm{a}$ at a temperature $T=1.5 \mathrm{~K} . k_{0}$ is determined by measurements of cantilever thermal motion at various temperatures and $V$ is determined through knowledge of the NW geometry from SEMs. The demagnetization factors are calculated according to Aharoni, ${ }^{29}$ making the approximation of a rectangular cross-section.

In most magnetometry measurements, we bring the system to a constant temperature $T$ and apply a large external field, $H$ $=1 \mathrm{~T}$, in order to magnetize the NW and initialize it in its fieldinduced ferromagnetic phase. We then sweep the magnetic field to $0 \mathrm{~T}$. In some cases, we cool the $\mathrm{NW}$ down through the critical temperature around $T=28 \mathrm{~K}$ with $H=0$, i.e., zero-field cooling (ZFC), in order to avoid memory effects from previous magnetic states. ${ }^{30}$ We find that ZFC gives different results from our conventional measurement procedure only at low fields $(|H|<0.1 \mathrm{~T})$ where hysteresis is observed. We therefore use ZFC in order to distinguish the transition between the helical and the conical phase, which is otherwise obscured by memory effects (see Supporting Information).

Note that the field-polarized ferromagnetic (FM) state remains unsaturated even at $H=6 \mathrm{~T}$ and $T=1.5 \mathrm{~K}$, as expected, and approaches a saturation magnetization within the measurement error for what is reported in bulk $\left(0.39 \mu_{B} / \mathrm{Mn}\right)$ and thin films $\left(0.42 \mu_{B} / \mathrm{Mn}\right)$ of $\mathrm{MnSi}^{31}$ (see Figure 2).

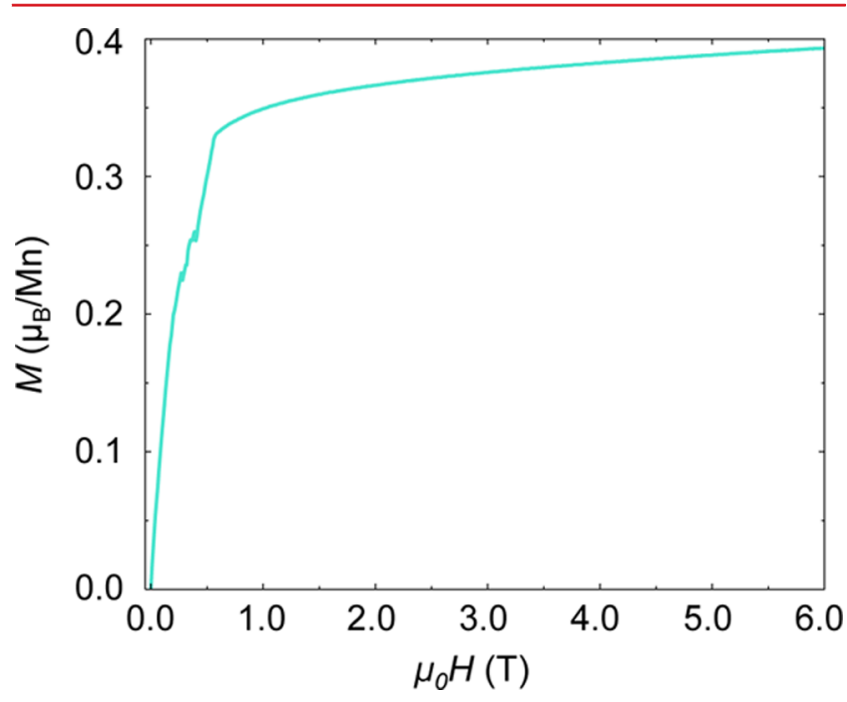

Figure 2. Behavior of magnetization at high field. $M(H)$ calculated from the measurements of $\Delta f(H)$ for NW1 at $T=9 \mathrm{~K}$, with the long axis parallel to the field. The field-polarized ferromagnetic state remains unsaturated even at $H=6 \mathrm{~T}$. It approaches a saturation magnetization close to what is reported for $\mathrm{MnSi}$ in bulk $\left(0.39 \mu_{B} /\right.$ $\mathrm{Mn})$ and thin films $\left(0.42 \mu_{\mathrm{B}} / \mathrm{Mn}\right)$.
Furthermore, $M(H)$ is characterized by a tilted plateau region as expected by Bauer and Pfleiderer for a sample with a skyrmion lattice phase. ${ }^{30}$ By fitting $M(H)$ and calculating $\partial M$ / $\partial H$, we assign the transition between the conical, skyrmion lattice, and ferromagnetic phases of the magnetic NW using similar procedures as Bauer and Pfleiderer (see Supporting Information). The sharp dips in $M(H)$ in Figure $3 \mathrm{~b}$ are fully reproducible and are robust to changes in the direction and sweep rate of the applied magnetic field. For now, we take note of the region in $H$ and $T$ where they appear and consider the possible physical mechanisms following the presentation of the data.

From measurements at temperatures between 0.4 and 31.8 $\mathrm{K}$, shown in Figure $3 \mathrm{~b}$, we obtain the phase diagram shown in Figure 4. The skyrmion lattice phase measured for NW1 extends from $T=28 \mathrm{~K}$ down to at least $0.4 \mathrm{~K}$ and stretches between $H \simeq 0.2$ and $0.5 \mathrm{~T}$. This region is significantly larger than the small pocket near the critical temperature observed in bulk $\mathrm{MnSi}$ (from $T=26$ to $28.5 \mathrm{~K}$ and $H=0.1$ to $0.25 \mathrm{~T}$ ) 4,30 and confirms the less direct magnetoresistance observations of $\mathrm{Du}$ et $\mathrm{al}^{20}$ and Liang et al. ${ }^{32}$ in this field geometry. Note that comparison between field-cooled (FC) and zero-field-cooled (ZFC) measurements also allows us to distinguish the transition between the helical and conical phase (see Supporting Information).

We make similar measurements on two more NW samples, NW2 and NW3. NW2, which is also mounted with its long axis parallel to $\mathbf{H}$, is a $26.7 \mu \mathrm{m}$ long $\mathrm{NW}$ with similar cross-sectional dimensions and grown on the same wafer as NW1. Measurements of $M(H)$ for NW2 show an extended skyrmion lattice phase similar to that measured in the shorter NW1 as well as a region of reproducible dips in $\Delta f(H)$, as is visible in Figure 3c.

In the third set of measurements we attach NW3 to the cantilever such that its long axis is aligned perpendicular to $\mathbf{H}$, as shown in Figure 1b. NW3 is a $7.8 \mu \mathrm{m}$ long segment of the same longer NW from which NW1 was cut and therefore, except for its length, is identical to NW1 (see Supporting Information). We plot $\Delta f$ as a function of $H$ in this configuration in Figure 5a for $T=27.5 \mathrm{~K}$. Because of the strong uniaxial shape anisotropy with the easy axis oriented perpendicular to $\mathbf{H}$, at low applied fields, the anisotropy energy overwhelms the Zeeman energy, forcing $\mathbf{M}$ to point along the long axis of the NW. Let us, for the moment, ignore the field range bounded by discontinuities around $|H|=0.2 \mathrm{~T}$. Excluding this range, $\Delta f$ is positive for $|H| \lesssim 0.3 \mathrm{~T}$ as is, therefore, the curvature of the magnetic energy $E_{m}$ with respect to the sample angle $\theta$. The sign of this curvature indicates that the direction of $\mathbf{M}$ in the sample is close to minimizing the anisotropy energy, i.e., $\mathbf{M}$ points nearly along the magnetic easy axis (long axis of $\mathrm{NW}$ ). As $\Delta f$ crosses zero and becomes negative (roughly $0.3<$ $|H|<0.4 \mathrm{~T}), \mathbf{M}$ points increasingly away from the easy axis and toward the hard axis (short axis of NW) where the anisotropy energy is maximized. This behavior corresponds to $\mathbf{M}$ tilting in the direction of $\mathbf{H}$. The sharp change in the slope of $\Delta f$ around $|H|=0.4 \mathrm{~T}$ reflects the coincidence of $\mathbf{M}$ with $\mathbf{H}$ and marks the transition from the conical to the field-polarized ferromagnetic state. $^{24}$ The subsequent gradual decrease in $\Delta f$ to more negative values results from the gradual increase of $\mathbf{M}$ along $\mathbf{H}$ as it approaches saturation. The diagram in the inset to Figure 5a schematically shows the progression of the net magnetization $\mathbf{M}$ inferred from $\Delta f(H)$ in NW3.

We now consider the field range bounded by the discontinuities in $\Delta f$ around $|H|=0.2 \mathrm{~T}$. These discontinuities 

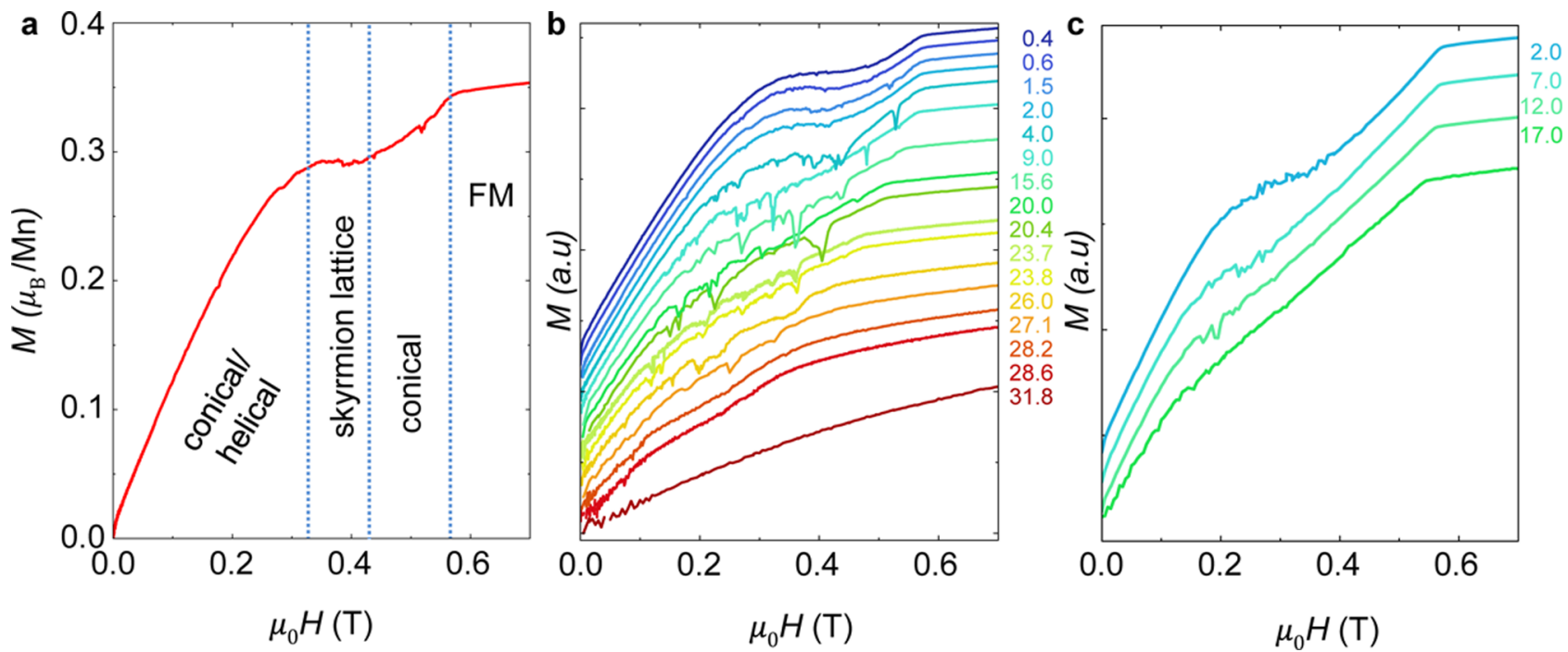

Figure 3. Dependence of magnetization on magnetic field with the NW parallel to the field. (a) $M(H)$ extracted from measurements of $\Delta f(H)$ for NW1 at $T=1.5 \mathrm{~K}$. The dashed blue lines mark transitions between the labeled magnetic phases. The transitions between conical/helical, skyrmion lattice, and field-induced ferromagnetic phase are determined by changes in $\partial M / \partial H$ (see Supporting Information). $M(H)$ for (b) NW1 and (c) NW2 measured at different temperatures (labeled on the right).

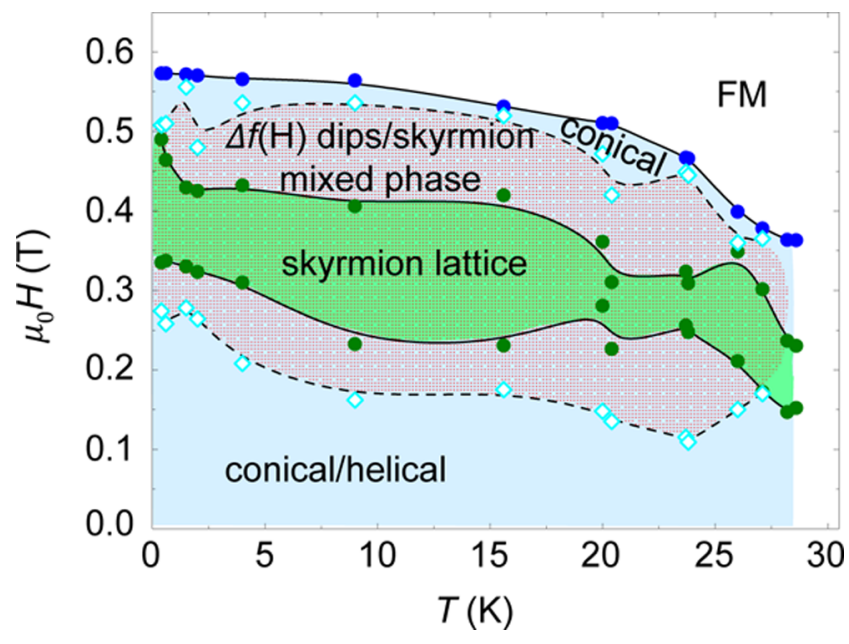

Figure 4. Phase diagram for MnSi NW parallel to the field. The magnetic phase diagram shows the boundaries between phases in $T$ and $H$ as determined from measurements of $M(H)$, as shown in Figure 3. Filled circles indicate the boundaries for the $7.1 \mu \mathrm{m}$ long NW1. Colored regions of the diagram serve as guides to the eye. The semitransparent red region denotes the region where the dips in $\Delta f(H)$ appear. The cyan squares indicate the first and the last dips as a function of $H$ observed in $\Delta f$.

indicate a first order phase transition and delineate a region in $T$ and $H$ similar to that known to correspond to the skyrmion lattice phase in bulk $\mathrm{MnSi}^{4,30}$ This phase is characterized by a reduction in $\Delta f$ with respect to the neighboring phases. This behavior is consistent with the reduction of $M$ associated with the formation of a skyrmion lattice in a previously conical phase. Measurements of NW3 in this geometry were repeated for temperatures between 2.0 and $29.0 \mathrm{~K}$, as shown in Figure $5 b$.

Sharp dips in $\Delta f(H)$ appear in a roughly equivalent range of $H$ and $T$ for the perpendicular geometry, e.g., Figure $5 b$, as for the parallel geometry, e.g., Figures $3 b, c$. Once again the features are fully reproducible and robust to changes in the direction and sweep rate of the applied magnetic field. We note that recent transport measurements by Liang et al. on $\mathrm{MnSi} \mathrm{NWs}$ from the same growth batch in the perpendicular magnetic field geometry show a THE signal in a similar range of $H$ and $T .{ }^{32}$ We postulate that this region corresponds to a mixture of skyrmion and conical or helical states, similar to that imaged by $\mathrm{Yu}$ et al. in LTEM measurements of $\mathrm{Fe}_{0.5} \mathrm{Co}_{0.5} \mathrm{Si}^{9}$ under perpendicular magnetic field. Such a phase would have spin chirality and would be expected to produce a THE signal. Note that the features always appear as dips in $\Delta f$, i.e., changes in $\Delta f$ toward more negative values. From eq 2 this trend indicates a process resulting in more negative curvatures of $E_{m}$ with respect to $\theta$, corresponding to a reduction in the angular magnetic confinement. The introduction of magnetic disorder would be consistent with these observations. Therefore, another possibility is that the features arise from domain wall motion within the NWs.

If we follow the interpretation of the data developed above, we obtain the phase diagram shown in Figure 6, suggesting a skyrmion lattice phase with an extent similar to that observed in bulk $\mathrm{MnSi}$ and therefore significantly reduced compared with that observed in NW1 and NW2 with their long axes aligned parallel to $\mathbf{H}$. Note that in NW3, the effect of shape-induced magnetic anisotropy appears in the position of the boundary between the conical and field-induced ferromagnetic state, shown in Figure 6. Compared to the phase diagram derived from $\mathbf{H}$ parallel measurements, shown in Figure 4, the transition fields in Figure 6 are shifted to higher values. The difference can be accounted for by calculating the much larger demagnetization field expected in the perpendicular geometry $\left(H_{d} \simeq 0.1 \mathrm{~T}\right)$ compared with that expected in the parallel geometry $\left(H_{d}<0.005 \mathrm{~T}\right)$.

The presented data indicate a stabilization of the skyrmion lattice phase that depends on the orientation of the NWs with respect to the applied magnetic field. Until now, two mechanisms have been proposed to explain experimental observations of skyrmion lattice phase stabilization. The first, based on spatial confinement effects, requires a sample dimension to be comparable or less than the skyrmion lattice constant $L_{D}{ }^{14}$ Since both the length and cross-sectional 
a

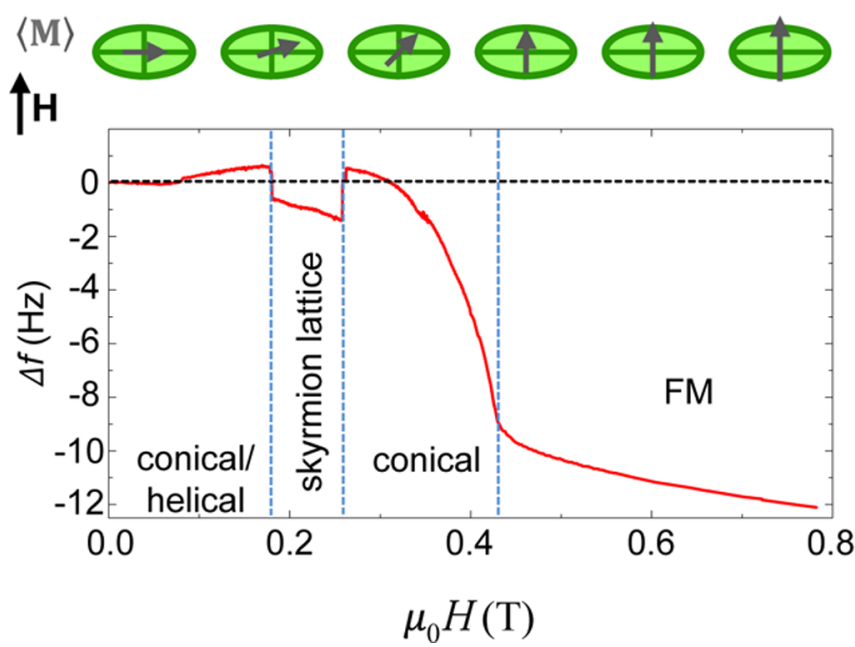

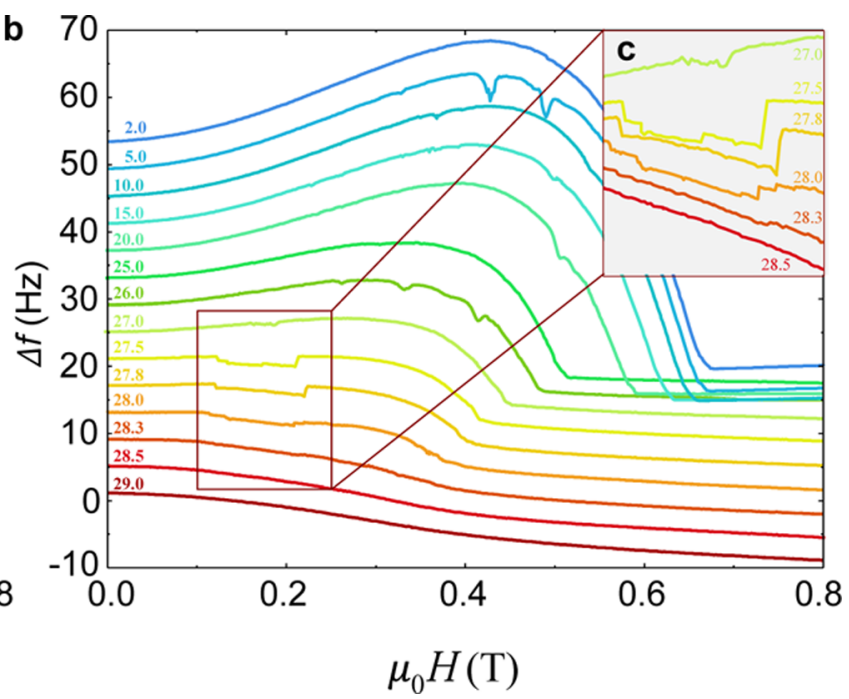

Figure 5. Dependence of frequency shift on magnetic field with the NW perpendicular to the field. (a) $\Delta f(H)$ for NW3 at $T=27.5 \mathrm{~K}$. The dashed blue lines mark transitions between the labeled magnetic phases. The transitions between conical/helical and skyrmion lattice and between skyrmion lattice and conical phases are determined by the sharp discontinuities in $\Delta f(H)$. The transition between the conical and field-induced ferromagnetic phase is determined by a sharp change in slope of $\Delta f(H)$. (b) $\Delta f(H)$ for NW3 measured at different $T$ between 2 and 29 K. For clarity, the $\Delta f(H)$ at different $T$ have been shifted by a constant frequency. (c) A detailed view of the data near the region of the skyrmion phase.

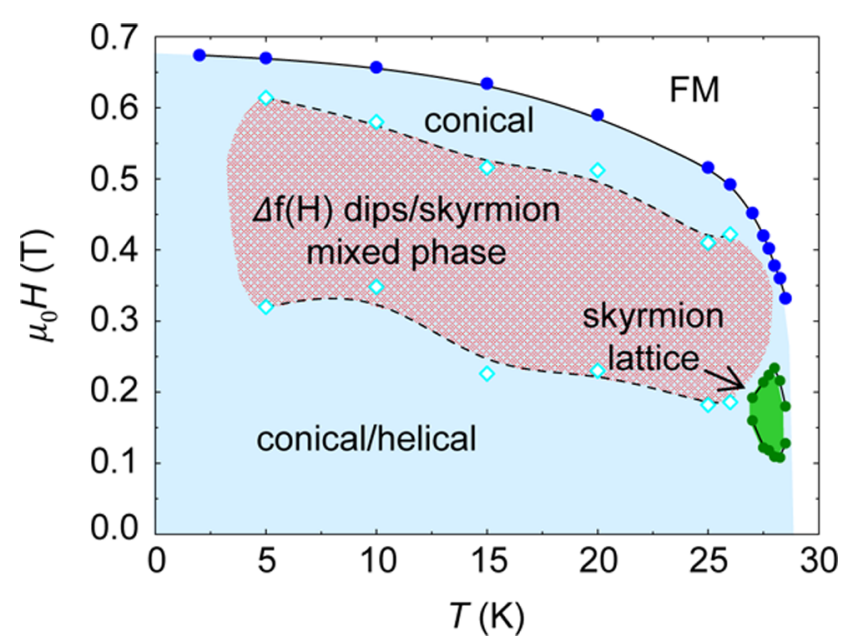

Figure 6. Phase diagram for a MnSi NW perpendicular to the field. The magnetic phase diagram shows the boundaries between phases in $T$ and $H$ as determined from measurements of $\Delta f(H)$, as shown in Figure 5. Filled circles delineate the phases measured for the $7.8 \mu \mathrm{m}$ long NW3. Colored regions of the diagram serve as guides to the eye. The semitransparent red region denotes the region where the sharp dips in $\Delta f(H)$ appear. The cyan squares indicate the first and last dips as a function of $H$.

dimensions of our NWs are much larger than $L_{D}=18 \mathrm{~nm}$ for $\mathrm{MnSi}$, we rule this mechanism out. The second mechanism requires a uniaxial magnetic anisotropy. ${ }^{15}$ For example, an easyaxis uniaxial anisotropy, combined with an applied magnetic field aligned parallel to the axis, can increase the energy of the conical phase due to its rotating off-axis magnetization components. As a result, the competing skyrmion lattice phase is stabilized. Uniaxial anisotropy can originate from shape, surface, interface, pressure, or crystal direction.

At this point it is instructive to recall that Bauer and Pfleiderer measured millimeter-scale single-crystal $\mathrm{MnSi}$ samples as a function of crystal orientation, shape, and field orientation, finding no evidence of skyrmion phase stabilization beyond the conventional bulk case. ${ }^{30}$ Magnetocrystalline anisotropy had a small effect on the extent of the skyrmion phase, likely due to its weakness in $\mathrm{MnSi}^{33}$ Shape anisotropy also had no stabilization effect, only producing a field shift in the phase diagram due to the demagnetization field. In particular, a $6 \times 1 \times 1 \mathrm{~mm}^{3}$ sample, whose demagnetization factors are similar to those of our NWs, showed no stabilization effect. The primary difference between these samples and our NWs is the size-scale and the large disparity in the surface-tovolume ratio that results. For long and narrow objects, the surface-to-volume ratio is dominated by surfaces whose normal is perpendicular to the long axis, making the ratio inversely proportional to the diameter. Our samples, with diameters on the order of $470 \mathrm{~nm}$, have surface-to-volume ratios several thousand times larger than the millimeter-scale samples of Bauer and Pfleiderer.

Therefore, we suggest that the demagnetization influence of these surfaces produces an effective magnetic anisotropy. ${ }^{26}$ Due to the DM interaction and missing spins near the boundary, ${ }^{34,35}$ spins at the surface align parallel to it. This effective anisotropy could suppresses the conical phase, which, combined with a parallel applied magnetic field localizing the skyrmion cores, would stabilize and extend the skyrmion lattice phase ${ }^{25}$ and perhaps produce a mixed skyrmion phase. Indications of a mixed skyrmion phase even in the perpendicular geometry may be attributed to the fact that, below the critical field, $\mathbf{M}$ is tilting toward $\mathbf{H}$ and maintains a component along the long axis of the NW. This coincidence of a component of $\mathbf{M}$ with the effective anisotropy axis could allow some stabilization of the skyrmion phase against the other phases. The stabilizing influence of boundaries on the skyrmion phase is observed in real-space measurements of skyrmion formation in FeGe thin-films, where skyrmions are seen to emerge from the helical phase near the sample edge and grain boundaries. ${ }^{10}$ One should also note the presence of the twinning boundary along the long-axis of the NWs produces an additional anisotropy. The DM interaction vanishes at this interface, leaving only ferromagnetic exchange. This additional boundary condition prohibits modulation of the magnetization along the twinning plane. 
In conclusion, we measure a series of high-aspect-ratio singlecrystal MnSi NWs and find strong evidence that their skyrmion phase can be stabilized and extended by an effective magnetic anisotropy. Measurements are made using sensitive dynamicmode cantilever magnetometry, which allows the investigation of individual nanometer-scale magnetic samples. The finding that an anisotropy, likely arising from the nanometer-scale geometry of the NWs, is enough to stabilize an extended skyrmion phase has important implications. In particular, such a stabilization mechanism would improve the viability of proposals for the use of skyrmions in thin magnetic wires as carriers of high-density information.

\section{ASSOCIATED CONTENT}

\section{S Supporting Information}

Mechanism behind the cantilever frequency shift, how to infer the magnetization from frequency shift, properties of the cantilevers and nanowire samples, determination of the phase transitions, and phase diagrams including the helical to conical transitions. The Supporting Information is available free of charge on the ACS Publications website at DOI: 10.1021/ acs.nanolett.5b02232.

\section{AUTHOR INFORMATION}

\section{Corresponding Authors}

*E-mail: xuef@hmfl.ac.cn.

*E-mail: jin@chem.wisc.edu.

*E-mail: martino.poggio@unibas.ch.

\section{Author Contributions}

F.X. conceived and designed the experiment with M.T., H.D., and M.P. The NWs were provided by D.L., M.J.S., and S.J. F.X. and A.M. prepared the samples and made the measurements. A.M., F.X., and M.P. analyzed the data. The interpretation was developed by A.M., F.X., D.L., S.J., and M.P. A.M. and M.P. wrote the manuscript. All authors discussed the results and contributed to the manuscript.

\section{Notes}

The authors declare no competing financial interest.

\section{ACKNOWLEDGMENTS}

The authors thank Dr. Dennis Weber and Dr. Boris Groß for technical support and helpful discussions. We acknowledge the support from the Canton Aargau, the Swiss National Science Foundation (SNF, Grant No. 200020-140478), the Swiss Nanoscience Institute, the National Center of Competence in Research for Quantum Science and Technology, the National Nature Science Foundation of China (NSFC, Grant No. 11374305 and 11174294), the U.S. National Science Foundation (NSF, Grant No. ECCS-1231916), and the National Key Basic Research of China (Grant No. 2010CB923403).

\section{REFERENCES}

(1) Dzyaloshinsky, I. J. Phys. Chem. Solids 1958, 4, 241.

(2) Moriya, T. Phys. Rev. 1960, 120, 91.

(3) Crépieux, A.; Lacroix, C. J. Magn. Magn. Mater. 1998, 182, 341.

(4) Mühlbauer, S.; Binz, B.; Jonietz, F.; Pfleiderer, C.; Rosch, A.; Neubauer, A.; Georgii, R.; Böni, P. Science 2009, 323, 915.

(5) Neubauer, A.; Pfleiderer, C.; Binz, B.; Rosch, A.; Ritz, R.; Niklowitz, P. G.; B”oni, P. Phys. Rev. Lett. 2009, 102, 186602.

(6) Pappas, C.; Leliévre-Berna, E.; Falus, P.; Bentley, P. M.; Moskvin, E.; Grigoriev, S.; Fouquet, P.; Farago, B. Phys. Rev. Lett. 2009, 102, 197202.
(7) Fert, A.; Cros, V.; Sampaio, J. Nat. Nano 2013, 8, 152.

(8) Yu, X. Z.; Kanazawa, N.; Zhang, W. Z.; Nagai, T.; Hara, T.; Kimoto, K.; Matsui, Y.; Onose, Y.; Tokura, Y. Nat. Commun. 2012, 3 , 988.

(9) Yu, X. Z.; Onose, Y.; Kanazawa, N.; Park, J. H.; Han, J. H.; Matsui, Y.; Nagaosa, N.; Tokura, Y. Nature 2010, 465, 901.

(10) Yu, X. Z.; Kanazawa, N.; Onose, Y.; Kimoto, K.; Zhang, W. Z.; Ishiwata, S.; Matsui, Y.; Tokura, Y. Nat. Mater. 2011, 10, 106.

(11) Tonomura, A.; Yu, X.; Yanagisawa, K.; Matsuda, T.; Onose, Y.; Kanazawa, N.; Park, H. S.; Tokura, Y. Nano Lett. 2012, 12, 1673.

(12) Huang, S. X.; Chien, C. L. Phys. Rev. Lett. 2012, 108, 267201.

(13) Li, Y.; Kanazawa, N.; Yu, X. Z.; Tsukazaki, A.; Kawasaki, M.; Ichikawa, M.; Jin, X. F.; Kagawa, F.; Tokura, Y. Phys. Rev. Lett. 2013, $110,117202$.

(14) Rybakov, F. N.; Borisov, A. B.; Bogdanov, A. N. Phys. Rev. B 2013, 87, 094424.

(15) Butenko, A. B.; Leonov, A. A.; Rößler, U. K.; Bogdanov, A. N. Phys. Rev. B 2010, 82, 052403.

(16) Stipe, B. C.; Mamin, H. J.; Stowe, T. D.; Kenny, T. W.; Rugar, D. Phys. Rev. Lett. 2001, 86, 2874.

(17) Parkin, S. S. P.; Hayashi, M.; Thomas, L. Science 2008, 320, 190. PMID: 18403702.

(18) Higgins, J. M.; Ding, R.; DeGrave, J. P.; Jin, S. Nano Lett. 2010, 10,1605 .

(19) Yu, X.; DeGrave, J. P.; Hara, Y.; Hara, T.; Jin, S.; Tokura, Y. Nano Lett. 2013, 13, 3755.

(20) Du, H.; DeGrave, J. P.; Xue, F.; Liang, D.; Ning, W.; Yang, J.; Tian, M.; Zhang, Y.; Jin, S. Nano Lett. 2014, 14, 2026.

(21) Tang, S.; Kravchenko, I.; Yi, J.; Cao, G.; Howe, J.; Mandrus, D.; Gai, Z. Nano Res. 2014, 7, 1788.

(22) Bleszynski-Jayich, A. C.; Shanks, W. E.; Peaudecerf, B.; Ginossar, E.; Oppen, F. v.; Glazman, L.; Harris, J. G. E. Science 2009, 326, 272. PMID: 19815772

(23) Jang, J.; Ferguson, D. G.; Vakaryuk, V.; Budakian, R.; Chung, S. B.; Goldbart, P. M.; Maeno, Y. Science 2011, 331, 186. PMID: 21233380

(24) Weber, D. P.; Ruffer, D.; Buchter, A.; Xue, F.; Russo-Averchi, E.; Huber, R; Berberich, P.; Arbiol, J.; Fontcuberta i Morral, A.; Grundler, D.; Poggio, M. Nano Lett. 2012, 12, 6139.

(25) Bogdanov, A. N.; Rößler, U. K. Phys. Rev. Lett. 2001, 87, 037203.

(26) Aharoni, A. Introduction to the Theory of Ferromagnetism, 2nd ed.; Oxford University Press: Oxford, 2001.

(27) Szczech, J. R.; Jin, S. J. Mater. Chem. 2010, 20, 1375.

(28) Rugar, D.; Mamin, H. J.; Guethner, P. Appl. Phys. Lett. 1989, 55, 2588.

(29) Aharoni, A. J. Appl. Phys. 1998, 83, 3432.

(30) Bauer, A.; Pfleiderer, C. Phys. Rev. B 2012, 85, 214418.

(31) Karhu, E. A.; Rößler, U. K.; Bogdanov, A. N.; Kahwaji, S.; Kirby, B. J.; Fritzsche, H.; Robertson, M. D.; Majkrzak, C. F.; Monchesky, T. L. Phys. Rev. B 2012, 85, 094429.

(32) Liang, D., DeGrave, J. P., Stolt, M. J., Tokura, Y., Jin, S. arXiv: 1503.03523 [cond-mat] (2015).

(33) Date, M.; Okuda, K.; Kadowaki, K. J. Phys. Soc. Jpn. 1977, 42, 1555.

(34) Du, H.; Ning, W.; Tian, M.; Zhang, Y. Phys. Rev. B 2013, 87, 014401.

(35) Leonov, A. O.; Rössler, U. K.; Mostovoy, M. EPJ Web of Conferences 2014, 75, 05002. 